\title{
Crónica de una muerte anunciada: La conveniencia de la arbitrabilidad de las patentes y marcas en el Perú
}

\author{
Paula Silva* \\ Adriana Ascue**
}

Resumen. - El presente artículo aborda alguno de los desafíos del arbitraje en materia de Propiedad Industrial. El análisis se centra específicamente en la arbitrabilidad de los conflictos derivados de la concesión de los derechos de marcas y patentes. A partir de un repaso de experiencias comparadas que han admitido la arbitrabilidad de estos derechos y de los distintos criterios de arbitrabilidad existentes, se examina si es conveniente arbitrar estas materias en el contexto peruano.

\begin{abstract}
This article deals with some of the challenges of industrial property arbitration. The analysis focuses specifically on the arbitrability of disputes arising from the grant of trademark and patent rights. Based on a review of comparative experiences that have admitted the arbitrability of these rights and the different arbitrability criterias, the authors examine whether it is convenient to arbitrate these matters in the Peruvian context.
\end{abstract}

Palabras claves. - Propiedad industrial, arbitrabilidad, orden público, marcas y patentes.

Keywords. - Industrial property, arbitrability, public policy, trademarks and patents.

\footnotetext{
* Estudiante de noveno ciclo de la Facultad de Derecho de la Universidad del Pacífico. Miembro de la Comisión de Comercialización de la Revista Forseti. Practicante en Bullard, Falla, Ezcurra +.

** Estudiante de noveno ciclo de la Facultad de Derecho de la Universidad del Pacífico. Subdirectora de la Comisión de Edición de la Revista Forseti. Asistente de investigación en el Centro de Investigación de la Universidad del Pacífico.
} 


\section{Introducción}

En los últimos años, debido al gran avance tecnológico de la informática y de otras tendencias como la biotecnología, la Propiedad Intelectual se ha visto afectada y ha aumentado exponencialmente ${ }^{1}$. Esto se ha visto reflejado, por ejemplo, en el incremento internacional de la explotación de patentes a través de acuerdos de licencia ${ }^{2}$. Esta creciente complejidad sumada a la internacionalización de la explotación de patentes ha puesto en evidencia las limitaciones del proceso tradicional de solución de controversias. Así, se vuelve imperiosa la implementación de un sistema que se adecue a las necesidades del mercado y pueda responder ante nuevas circunstancias técnicas.

Sin embargo, antes de profundizar más sobre este último punto, es menester abordar algunos términos que serán recurrentes a lo largo de esta investigación. La Propiedad Intelectual es definida por la Organización Mundial de la Propiedad Intelectual (OMPI) ${ }^{3}$ como "las creaciones de la mente, obras literarias y artísticas, así como símbolos, nombres e imágenes utilizados en el comercio". Esta rama del Derecho está dividida en la mayor parte de legislaciones en dos grandes subgrupos: los Derechos de Autor y la Propiedad Industrial.

El primero, más conocido por su traducción en inglés copyright, comprende la protección de la forma en que los autores han plasmado sus ideas, pensamientos literarios y artísticos. Es importante mencionar que el objeto de protección es la forma de la expresión, más no la idea en sí. Esta salvaguarda a este derecho implica el impedimento de plagio o de reproducción de la obra sin el consentimiento del autor. Este es un derecho declarativo, pues no se requiere que el titular de la obra haga algún trámite o esté constatado en algún registro para que éste exista.

Por otro lado, la Propiedad Industrial engloba principalmente a los derechos sobre las nuevas creaciones (patentes) y a los derechos de marcas. Estos han sido abordados por distintos dispositivos internacionales como el Convenio de París para la Protección de la Propiedad Intelectual enmendado en 1979, el cual señala que:

“La protección de la propiedad industrial tiene por objeto las patentes de invención, los modelos de utilidad, los dibujos o modelos industriales, las marcas de fábrica o de comercio, las marcas de servicio, el nombre comercial, las indicaciones de procedencia o denominaciones de origen, así como la represión de la competencia desleal"4.

1 LIU, Deming. Think twice before arbitrating a patent dispute?. En The American Review of International Arbitration, vol. 23, núm. 2, 2012, p.1.

2 Idem

3 Organización Mundial de la Propiedad Intelectual. (Publicación 450-S). ¿Qué es la Propiedad Intelectual?. Consultado el 10 de julio de 2019 en: https://www.wipo.int/edocs/pubdocs/es/intproperty/450/wipo_pub_450.pdf

4 Convenio de París sobre la Protección de la Propiedad Industrial 
(Énfasis agregado).

Como puede observarse, la Propiedad Industrial está compuesta por una gama de derechos con diversa naturaleza jurídica. Por un lado, están los derechos de marcas, patentes y modelos industriales, que suponen la concesión por parte del Estado al privado de derechos patrimoniales que le otorgan al titular la potestad de exclusión de terceros sobre su uso comercial. Por otro lado, se encuentra un derecho negativo de limitación de conductas: la represión de conductas desleales. Este a diferencia del resto no es objeto de ningún derecho patrimonial, sino de un derecho de exclusión más acotado porque "solo pueden ejercerse contra una clase limitada de infractores y no pueden venderse o negociarse de la forma habitual en que lo hacen los derechos de propiedad"5.

Esta rama, a diferencia de la primera, comprende derechos constitutivos. En otras palabras, para estos casos sí es necesario que el autor registre su derecho para que pueda gozar de protección. Una vez concedida al titular el derecho sobre la patente, este gozará del monopolio de su administración por un periodo largo de tiempo. Para efectos de este artículo, solo se analizará lo concerniente a patentes y marcas dentro del amplio universo de derechos de la Propiedad Industrial.

Así, en este artículo se responderá a la interrogante sobre si es conveniente para el Perú la arbitrabilidad en materias de patentes y marcas. A partir de un análisis de la jurisprudencia y la doctrina, se delimitarán los alcances de la materia arbitrable y finalmente se concluirá si resultaría idónea para Sistemas Jurídicos como el peruano o si sería una crónica de una muerte anunciada.

Antes de proceder a desglosar las aristas de esta problemática, es menester mencionar que existen tres distintos conflictos que se derivarían del derecho de patentes y marcas: i) respecto de la explotación de estos derechos, ii) respecto de la titularidad de estos derechos, y iii) respecto de la validez de la concesión de estos derechos. Esta acotación será relevante cuando se analice específicamente el acápite III: la aplicación de los criterios aplicables a la arbitrabilidad.

\section{Beneficios de un mecanismo de solución alternativa en la Propiedad}

\footnotetext{
Artículo 1

1) Los países a los cuales se aplica el presente Convenio se constituyen en Unión para la protección de la propiedad industrial.

2) La protección de la propiedad industrial tiene por objeto las patentes de invención, los modelos de utilidad, los dibujos o modelos industriales, las marcas de fábrica o de comercio, las marcas de servicio, el nombre comercial, las indicaciones de procedencia o denominaciones de origen, así como la represión de la competencia desleal.

3) La propiedad industrial se entiende en su acepción más amplia y se aplica no sólo a la industria y al comercio propiamente dichos, sino también al dominio de las industrias agrícolas y extractivas y a todos los productos fabricados o naturales, por ejemplo: vinos, granos, hojas de tabaco, frutos, animales, minerales, aguas minerales, cervezas, flores, harinas.

4) Entre las patentes de invención se incluyen las diversas especies de patentes industriales admitidas por las legislaciones de los países de la Unión, tales como patentes de importación, patentes de perfeccionamiento, patentes y certificados de adición, etc.

5 GRANTHAM, William. "The Arbitrability of International Intellectual Property Disputes". En Berkeley Journal of International Law, vol. 14, núm. 1, 1996.
} 


\section{Intelectual}

Si bien el arbitraje en sí mismo provee a las partes de distintos beneficios como la celeridad y la confidencialidad, los beneficios de su uso incrementan específicamente en materia Propiedad Intelectual. En este sentido, podemos mencionar tres grandes beneficios de la institución arbitral: (i) posibilidad de elección de la legislación aplicable al fondo, (ii) el peligro de litigios paralelos, pronunciamientos divergentes y falta de neutralidad; y (iii) la formación de un tribunal especializado. Cada uno de estos puntos será abordado en las siguientes líneas.

\section{i. Posibilidad de elección de la legislación aplicable al fondo}

Una primera dificultad inherente a los litigios de Propiedad Intelectual es la falta de unificación latinoamericana respecto a la regulación de la misma. Si bien la suscripción a distintos Tratados de Libre Comercio brinda una luz a la posibilidad de unificación, el silencio y las ambigüedades que los caracterizan marcan la necesariedad de la legislación y jurisprudencia nacional como complemento ${ }^{6}$. Además, pese a que los Estados tienen la potestad de incorporar a su legislación cualquier elemento que conviva con los tratados, ello dificulta la unificación de normas en la región sobre Propiedad Intelectual.

Siendo este el escenario internacional en el que se desenvuelven distintos contratos sobre propiedad industrial, la impredictibilidad sobre cuál sería la norma sustantiva que resuelva algún potencial conflicto producto del contrato representa una problemática real en esta materia. Una solución dirigida hacia la unificación de las legislaciones sería utópica y poco viable, por lo que resulta necesario un método de solución alternativo en el que las partes puedan pactar la norma aplicable al fondo de la controversia. Es allí que la posibilidad del arbitraje como método alternativo de solución de controversias se presenta como una opción viable y beneficiosa para las necesidades de este mercado.

\section{ii. El peligro de litigios paralelos y pronunciamiento divergentes}

La impredictibilidad sobre la norma aplicable no es el único problema respecto a la dependencia de determinadas normas nacionales, sino también la determinación de la jurisdicción competente. Desde la tradición romano germánica y el civil law existen distintas formas de concebir la jurisdicción 7, estas diferencias aumentan entre los distintos países seguidores de estas tradiciones, la aplicación de criterios característicos de una sola de estas corrientes como el de forum non conveniens y otros criterios. Esta potestad jurisdiccional abre la posibilidad de que más de una jurisdicción se declare competente y la posible

6 DÍAZ PÉREZ, Álvaro. América Latina y el Caribe: La propiedad intelectual después de los tratados de libre comercio. Chile: Naciones Unidas, 2008, pp. 242.

7 GABUARDI, Carlos. "Entre la jurisdicción, la competencia y el forum non conveniens". En Boletín mexicano de Derecho Comparado, núm. 121, 2008, p. 75. 
generación de pronunciamientos divergentes.

Aún en el caso de que la conexión con una jurisdicción sea evidente, ello no evita un problema de neutralidad. La neutralidad es relevante para la resolución de litigios sobre denominación de origen, marcas o signos distintivos podrían incluir partes de distintos países, y en este escenario la posibilidad de que la jurisdicción competente favorezca al nacional representa una gran problemática para los litigios como una opción viable de resolución de conflictos sobre Propiedad Industrial. En este escenario resulta necesario una forma alternativa de resolución de conflictos. De acuerdo a Fernández Rosas, el arbitraje brinda un escenario de neutralidad para la resolución de conflictos internacional:

"Si bien por el momento no cabe hablar propiamente de «anacionalidad», la neutralidad que proporciona el arbitraje, a partir del protagonismo jurídico de la sede donde se desarrolla, constituye un elemento psicológico de extraordinaria importancia en favor del juicio de árbitros" ${ }^{\prime \prime}$.

\section{iii. Formación de un tribunal especializado}

Otra problemática inherente a cualquier litigio de Propiedad Industrial es la falta de especialización de los tribunales nacionales. Las controversias sobre Propiedad Intelectual podrían versar sobre materia comercial, y por ende, dislumbrarse en un juzgado comercial. Sin embargo, las particularidades de la materia no permiten que un juez comercial pueda comprender o analizar de manera técnica dicho conflicto. En este escenario el arbitraje nuevamente brinda una opción viable pues:

"En el caso del arbitraje o la mediación, los cuales podría tratar sobre asuntos técnicos bastante complejos, las partes pueden nombrar a individuos versados en el tema, con la experiencia y el conocimiento necesarios para llegar a una solución mutuamente satisfactoria. Más aún, es poco probable que en caso el asunto se ventilara ante los tribunales, estos tuvieran la necesaria capacidad técnica para resolver el asunto debidamente" ${ }^{\prime \prime}$.

\section{Experiencia comparada}

Alrededor del mundo existen distintas legislaciones que han amparado la posibilidad de arbitrar la Propiedad Intelectual. A modo de ilustración, mencionaremos tres casos emblemáticos: Bélgica, EE.UU y Suiza. Bélgica ha establecido en su ordenamiento jurídico que cualquier materia puede ser sometida a arbitraje salvo los asuntos que contravengan las leyes y/o normas mismas $^{10}$. El arbitraje belga permite de manera expresa el arbitraje respecto a los conflictos derivados del derecho de patentes y marcas. En esta misma línea, la

8 FERNÁNDEZ ROZAS, Jose. “Determinación del lugar de arbitraje y consecuencias del control del laudo por el tribunal de la sede arbitral". En Lima Arbitration, núm. 2, 2007, p. 60.

9 FERRERO DIEZ CANSECO, Gonzalo. "Medios alternativos de solución de controversias y propiedad intelectual”. En Ius et veritas, vol. 19, 1999, p. 104.

10 GRANTHAM, Op. cit. , p. 14. 
Corte Suprema belga ha señalado que si bien la validez de las patentes es un asunto de interés general, no es de interés público ${ }^{11}$, por lo que no incurriría en un supuesto de falta de arbitrabilidad.

El segundo caso es el de Estados Unidos que también permite la arbitrabilidad de los conflictos de validez en patentes. El laudo que resuelva esta controversia solo será vinculante entre las partes y "las partes podrán acordar que el laudo arbitral se modifique cuando un tribunal adopte posteriormente una decisión definitiva sobre la validez o ejecutoriedad de la patente"12. Sin embargo, en dicho sistema no se encuentra regulado lo referente a las marcas comerciales. No obstante a dicha limitación, los tribunales americanos consideran de manera positiva el arbitraje de marcas siempre y cuando se manifieste de manera extensa en donde puedan calzar los parámetros establecidos por la legislación anteriormente mencionada.

Finalmente, en el caso de Suiza, todo aspecto relacionado a la propiedad puede ser llevado a arbitraje según su legislación vigente. Ello incluye de manera expresa a la Propiedad Intelectual. Es menester resaltar que dicha disposición es posible de realizar siempre y cuando no se contravenga el orden público ni materias impuestas a disposición por el Estado ${ }^{13}$.

A pesar de que el arbitraje en estos países no ha suscitado mayores controversias, la arbitrabilidad de los conflictos de validez y titularidad de patentes y marcas, esta tendencia no se ha generalizado en el resto del mundo.

\section{Criterios aplicables de arbitrabilidad}

Habiendo delimitado las distintas ramas de la Propiedad Intelectual, los posibles beneficios del uso del arbitraje en estas y cómo otras legislaciones lo han adoptado en su ordenamiento, resulta necesario determinar la posibilidad de su uso. Si bien existen distintos criterios internacionales para responder a la pregunta de qué puede someterse a arbitraje, existen materias que han generado cierta dificultad para ser ubicadas en esta clasificación, entre ellas: la Propiedad Industrial14. Si bien la dependencia del orden público nacional para la determinación de la materia arbitrable objetiva dificulta la generación de un consenso internacional respecto a su definición ${ }^{15}$, se han llegado a aproximaciones de su definición o a fórmulas legislativas que lo definen.

Ibid., p. 20.

13 Swiss Chambers Arbitration Institution. Consultado el 15 de julio de 2019 en: https://www.swissarbitration.org/files/33/Swiss-Rules/SRIA_EN_2017.pdf

14 VÁSQUEZ PALMA, Maria Fernanda. "La arbitrabilidad objetiva: Aspectos históricos, modernas tendencias en derecho comparado y ubicación en el escenario legislativo chileno". En Ius et Praxis, vol. 12, núm. 1, 2006, pp. 181-213.

15 Idem. 
Las distintas fórmulas legislativas apuntan hacia la arbitrabilidad en aquellos casos en los que se disputen derechos de libre disposición. De acuerdo a Lorca Navarrete y Silguero Estagnan, este criterio principal implica que:

“Los términos poder de disposición - libre disposición son [...] aquellas sobre las que las partes están facultadas para ejercer su autonomía de voluntad con la válida creación, extinción, modificación o configuración de relaciones jurídicas, esto es, la potestad normativa creadora que, a través del negocio jurídico, expresión de ese poder o libertad y fuente de disposiciones, reglamentaciones o normas privadas, el ordenamiento reconoce y ampara [...], en tanto no recaiga en materia sustraída a la autorregulación de los particulares, ya de todos -indisponibilidad absoluta. $[\ldots]^{\prime \prime 16}$

Para analizar si los derechos derivados de la Propiedad Industrial se encuentran dentro de este supuesto de libre disponibilidad resulta necesario recordar los tres tipos de posibles conflictos adelantados en la introducción derivados de la Propiedad Industrial: i) respecto de la explotación de estos derechos, ii) respecto de la titularidad de estos derechos; y, iii) respecto de la validez de la concesión de estos derechos.

Existe un cierto consenso respecto a que las controversias sobre explotación de un derecho sean arbitrables pues derivan de una relación contractual entre dos privados, quienes tienen la potestad de crear, modificar o extinguir dicha relación contractual. Es decir, cumplen con lo descrito por el criterio de libre disponibilidad. Adicionalmente a ello, de acuerdo a De Miguel Asensio, esta es la aplicación más típica pues el arbitraje requiere de una cláusula arbitral que lo active, y para ello se encuentra como prerrequisito un contrato.

“La práctica demuestra que típicamente el arbitraje se encuentra vinculado a la explotación contractual de estos derechos, en la medida en que el convenio arbitral que es presupuesto del arbitraje suele nacer en conexión con un contrato, con gran frecuencia como una cláusula más del contrato con respecto al cual las partes acuerdan que las diferencias que puedan surgir sean resueltas a través de esa vía"17.

Sin embargo, en la práctica esta separación ideal entre estos varios tipos de conflictos no siempre se cumple, pues podría ocurrir que pese a que el arbitraje se inicie sobre determinado conflicto respecto a la explotación contractual de algún derecho, sea necesario para el tribunal arbitral pronunciarse respecto a la validez o titularidad del derecho. En aquellos casos Hanotion señala que:

“Habida cuenta de la tendencia a la expansión de la arbitrabilidad, existe una propensión extendida a aceptar que el órgano arbitral pueda pronunciarse, si bien únicamente con carácter incidental o eficacia inter

16 LORCA NAVARRETE, Antonio María y SILGUERO ESTAGNAN, Joaquín. Derecho de Arbitraje Español. España: Dykinson, 1994, p. 38.

17 DE MIGUEL ASENSIO, Pedro. "Alcance de la arbitrabilidad de los litigios sobre derechos de propiedad industrial”. En Revista de Arbitraje Comercial y de Inversiones, vol. 7, núm. 1, 2014, p. 83 
partes acerca de la validez del derecho de propiedad industrial, en litigios relativos a la infracción de tales derechos o a contratos relativos a su explotación, sin que ello suponga ir más allá de lo arbitrable"18.

Vemos cómo esta interpretación no transgrede al interés público pues al tener un efecto únicamente inter partes y como finalidad principal la determinación de un conflicto entre privados, no excede las potestades del arbitraje ni constituye un derecho cuya constitución erga omnes corresponde únicamente al Estado. Ello concuerda con el pronunciamiento de la Cámara de Comercio Internacional (CCI) en la decisión $\mathrm{N}^{\circ}$ 6097. Esto, pues al darse una controversia durante el arbitraje sobre la validez de una patente el tribunal señaló que solo un tribunal nacional con la jurisdicción adecuada podría invalidar una patente erga omnes. Sin embargo, ello no impidió la continuación del arbitraje pues el tribunal sí se consideró facultado para confirmar si el demandante puede fundamentar las alegaciones basadas en sus patentes a pesar de las objeciones del demandado ${ }^{19}$.

Este aporte es sustancial para el éxito del arbitraje de explotación de un derecho industrial pues si el árbitro se declarará incompetente con la sola objeción de inexistencia del derecho de patente, esta objeción se instrumentalizaría como una forma efectiva de huir al arbitraje pactado entre las partes.

Pese a esta equilibrada solución, el arbitraje sobre la explotación de derechos de marca o patente tiene aún algunos rezagos públicos que dificultan su posterior ejecución. Pese a que estos contratos están dotados de un carácter privado, es innegable que el marco regulador que los rodea (regulación de competencia y derechos de consumidor) es considerado de interés público y contiene una considerable cantidad de normas imperativas, es así que resulta preocupante los posibles efectos de ello en la determinación de competencia del tribunal arbitral o en el posterior reconocimiento del laudo. Sobre ello la doctrina ha señalado que:

“En relación con los contratos de explotación de derechos de propiedad industrial, la circunstancia de que puedan resultar aplicables normas de defensa de la competencia, por ejemplo, relevantes de cara a la eventual nulidad de ciertas estipulaciones contenidas en contratos de transferencia de tecnología o para fijar el resarcimiento de daños y perjuicios, no afecta a la arbitrabilidad de las controversias, como ha puesto relieve la reciente práctica judicial española" 20 .

Una vez consolidada esta doctrina se esclarecerá la situación de la explotación de marcas o patentes respecto al arbitraje pues se le impediría al Poder Judicial la anulación de laudos por la sola presencia de normas imperativas relacionadas a la materia arbitrable.

18 HANOTIAU, Bernard. “L'arbitrabilité des litiges de propriété intellectuelle: une analyse comparative". En DE WERRA, Jacques, La résolution des litiges de propriété intellectuelle, Ginebra: Schulthes, 2010, pp. 155-174, pp. 160-162.

19 GRANTHAM, Op. cit., p. 8.

20 DE MIGUEL ASENSIO, Op. cit., p. 88. 
Si bien la arbitrabilidad de los derechos de explotación ya parece ser una doctrina adoptada por la comunidad internacional y materializada en distintas legislaciones, aún no existen demasiadas luces respecto al destino de los conflictos respecto de la validez o titularidad de estos derechos. Estos no se encuentran dentro del supuesto de libre disposición pues es el Estado, con efecto erga omnes, quien determina la existencia y titularidad de marcas y patentes. Siendo ello así, para determinar la posibilidad de arbitrabilidad de estos conflictos resulta necesario realizar un análisis más profundo respecto al concepto de materia arbitrable objetiva.

La materia arbitrable objetiva se presenta como un límite a la autonomía de la voluntad cuyo fundamento se encuentra en el interés general, ello bajo dos tendencias distintas de evaluación: (i) una arbitrabilidad contractual, bajo la cual la materia susceptible a arbitraje viene dada por el convenio arbitral; $y$, (ii) una arbitrabilidad procesal mediante la cual podrán ser susceptibles a arbitraje aquellas materias que no se encuentren reguladas por normas imperativas ${ }^{21}$. Es decir, altamente relacionada al orden público.

En este escenario, el planteamiento de la política pública de abrir mediante ley la posibilidad de arbitrar dichos conflictos dependerá entonces del enfoque brindado por el Estado respecto a si depende del reparto de competencias que realiza un Estado o a la viabilidad o practicidad de su uso en determinada materia 22 . De acuerdo a Bouza, pese a la potestad restrictiva de los Estados sobre la materia arbitrable, existe una tendencia de ampliación de las materias susceptibles:

"Por un lado, el arbitraje aparece rígidamente enmarcado por exigencias derivadas de otros sectores del ordenamiento, en lugar de responder a exigencias derivadas de su naturaleza propia y específica. Por el otro, la práctica ha evolucionado en sentido contrario a los resultado restrictivos al que conduce este planteamiento, ampliando progresivamente las materias susceptibles de arbitraje" 23 .

Esta evolución menos restrictiva cobra sentido con la tesis minimalista del orden público descrita por González de Cossio, de acuerdo a lo siguiente:

"Mientras que los primeros postulan una concepción amplia del orden público internacional, que incluya todas las leyes de policía, así como un control profundo de los laudos para verificar que el orden público aplicable está siendo respetado, lo cual conlleva una revisión de fondo de los laudos; la minimalista considera que, aún en la presencia de leyes de policía o principios de orden público, el control de laudo por el juez de nulidad o

21 CHILLÓN Y MEDINA, Jose María y MERINO MERCHÁN, Jose. Tratado de arbitraje privado interno e internacional. Madrid: S.L. Civitas Ediciones, 1991, p. 648.

22 Idem.

23 BOUZA VIDAL, Nuria. "La arbitrabilidad de los litigios en la encrucijada de la competencia judicial internacional y de la competencia arbitral". En Revista española de Derecho Internacional. vol. 52, núm. 2, 2000, p. 373 
ejecución debe ser mínimo y no puede anular o no ejecutar el mismo más que en casos excepcionales" 24 .

Pese a que Gonzales de Cossio hace referencia a la ejecución y reconocimiento de laudos, este mismo criterio es aplicable para la delimitación de la materia arbitrable objetiva pues la lógica detrás de este es que la sola presencia de normas de orden público o imperativas no implican la imposibilidad de acción del arbitraje en un conflicto, esta intervención arbitral será ilegítima únicamente cuando efectivamente se hayan violentado o transgredido dichos principios. Declarar como materia no arbitrable los conflictos sobre existencia o titularidad de marcas y patentes únicamente por la presencia de normas imperativas o de orden público, bajo esta lógica, no sería una restricción válida pues únicamente debería sujetarse a un control posterior de verificar que no se hayan vulnerado estos principios durante el proceso. Esta es la opción propuesta por Vásquez Palma:

"Por lo anterior, se ha postulado la idea de ampliar el espectro de las materias arbitrables, incluso aquellas "sensibles" (en tanto susceptible de afectar intereses generales o de terceros), en el entendido de que el riesgo de que un árbitro pronuncie una sentencia contraria al orden público no debiera conjugarse negando la arbitrabilidad sino en todo caso intensificando el control judicial del laudo"25.

Estando a ello, resulta lógico afirmar que el criterio de libre disponibilidad no es el único bajo el cual se puede declarar como arbitrable determinada materia. El Estado podría mediante ley declarar arbitrable determinada materia pese a no ser disponible. Habiendo establecido esta potestad, resulta necesario realizar un análisis de conveniencia de esta política pública aterrizado al Perú.

\section{La conveniencia de la arbitrabilidad de derechos de patente y marcas en el Perú}

En el Perú los procedimientos arbitrales tanto nacionales como internacionales se rigen por el Decreto Legislativo 1071, Ley de Arbitraje. Esta ley determina que las materias susceptibles de arbitraje (arbitrabilidad objetiva) son "las controversias sobre materias de libre disposición conforme a derecho, así como aquellas que la ley o los tratados o acuerdos internacionales autoricen"26. A partir de este artículo se desprenden dos componentes de la arbitrabilidad objetiva en el Perú. El primero, ya desarrollado en el acápite anterior, supone la posibilidad de someter a arbitraje las controversias de materias que sean de libre disposición. El segundo criterio que puede derivarse de este artículo es que aquellas materias

24 GONZÁLEZ DE COSSIO, Francisco. “Orden público y arbitrabilidad: dúo-dinámico del arbitraje". En Revista Auctoritas Prudentium, núm. 1, 2008, p. 10

25 VÁSQUEZ PALMA, Op. Cit, pp. 181-213.

26 Decreto Legislativo 1071, Ley de Arbitraje Artículo 2.- Materias susceptibles de arbitraje.

1. Pueden someterse a arbitraje las controversias sobre materias de libre disposición conforme a derecho, así como aquellas que la ley o los tratados o acuerdos internacionales autoricen. 
autorizadas por ley podrán también ser susceptibles de arbitraje.

Entonces, de acuerdo a lo analizado previamente la arbitrabilidad de los conflictos sobre la explotación de los derechos de Propiedad Industrial podría ser aceptaba por ser de libre disponibilidad de las partes. Sin embargo, cuando el conflicto deviene en la determinación de la titularidad o validez del derecho en cuestión, sólo cabría la posibilidad de someterlo a arbitraje si una ley lo autoriza expresamente, tal como es el caso de la expropiación. El principal problema que salta a la vista y que precisamente es objeto de esta investigación es si, teniendo en consideración las particularidades del caso peruano, es conveniente autorizarlo a través de una ley. Luego del análisis realizado se han hallado las siguientes problemáticas para la aplicación en el caso peruano.

\section{i. Problema de ejecución}

Como ya ha sido analizado previamente, la inscripción o registro de marcas y patentes depende únicamente del Estado, por lo que incluso si arbitralmente se laudara sobre la existencia o titularidad de un derecho de Propiedad Industrial, corresponde al Estado ejecutar esta decisión. Esto presenta una primera problemática pues es el mismo Estado, el cual podría no desear el reconocimiento y ejecución de este laudo, quien tiene la posibilidad de realizar el control posterior de esta decisión.

¿Cómo podría anularse o no reconocerse un laudo si mediante ley de manera expresa se ha habilitado la materia arbitrable? Lo cierto es que, como se ha tratado a lo largo de la investigación, existen diversas normas imperativas alrededor de la Propiedad Industrial, como la regulación sobre competencia o de protección al consumidor. La presencia de dichas normas da pie a una posible anulación debido a que en la exhaustiva revisión que realizan los jueces peruanos podrían considerar que no se aplicó una norma imperativa pertinente o que esta no fue interpretada correctamente.

Incluso en el arbitraje internacional en el que el reconocimiento y ejecución de laudos internacionales se rigen por la Convención de Nueva York de 1958, el Estado peruano podría negarse al reconocimiento yejecución. En este instrumento convencional se establece en su artículo $\mathrm{V}$ las causales taxativas para la denegación de reconocimiento y ejecución de laudos extranjeros. Dentro de estas, se establece que un Estado podrá negarse cuando la autoridad competente comprueban dos supuestos: (i) según la ley de ese país, el objeto de la diferencia no es susceptible de solución por vía de arbitraje; o (ii) cuando el reconocimiento o la ejecución de la sentencia serían contrarios al orden público de ese país.

Respecto del primer criterio, deberá recurrirse nuevamente a lo señalado por el artículo 2 de la Ley de Arbitraje del Perú y analizar los dos elementos que lo componen, y en el hipotético en el que los conflictos de titularidad y validez hayan sido expresamente autorizados por ley, podría concluirse que los 
conflictos superan con éxito el primer criterioEntonces, si la ley autoriza que esto sea arbitrable, ¿por qué motivo el Perú podría negarse a ejecutar un laudo? Como ya se ha señalado, la Propiedad Industrial está rodeada de normas imperativas, lo cual en países como el nuestro resulta bastante peligroso para el arbitraje. El Poder Judicial peruano podría considerar que la sola presencia de normas imperativas en el caso, sería vulneratorio del orden público.

Siendo este un escenario en el que la ejecución del laudo es en extremo necesaria y esta depende del Estado debido al uso del poder de policía, la posibilidad de que este coloque trabas a la ejecución es preocupante. Resulta lógico que las partes prefieran acudir de manera directa al Estado, por ello el arbitraje terminaría por inutilizarse.

\section{ii. La incorrecta interpretación de algunos criterios para la determinación de la competencia}

Si bien es cierto el árbitro es competente para determinar su propia competencia, ello no resulta suficiente en este caso para evitar caer ante el Poder Judicial peruano. Como hemos visto en el acápite de criterios aplicables a la arbitrabilidad, existen dos puntos sustanciales que el árbitro debe tomar en cuenta para la determinación de su competencia: (i) la existencia de normas imperativas dentro del conflicto no determinan su incompetencia; y, (ii) la objeción de inexistencia de derecho no impiden un pronunciamiento sobre la explotación del mismo.

Como bien señala De Miguel Asensio en lo desarrollado en el acápite IV, la existencia de normas imperativas o de orden público dentro del conflicto no afecta la arbitrabilidad de la materia, y por ende, tampoco la competencia del árbitro para pronunciarse sobre ella. El alto riesgo de error en esta interpretación o una interpretación distinta genera la inutilidad del pacto arbitral en este caso, pues la probabilidad de la aparición de normas imperativas es bastante alta.

De la misma forma el criterio establecido por la CCI de continuar con la controversia sobre explotación pese a la aparición de unaobjeción sobre su existencia es relevante para el éxito del arbitraje en esta materia pues de lo contrario, la parte demandada podría utilizar la misma como una forma de huir al arbitraje. Determinando así, nuevamente, la inutilización de la vía arbitral.

Estos criterios técnicos desarrollados por la doctrina internacional son ajenos a la doctrina y jurisprudencia peruana, por lo que la posibilidad de que no sean utilizados representa un grave peligro al arbitraje en esta materia.

Es decir, si bien, jurídicamente podría caber la posibilidad de que todos los conflictos derivados de los derechos de patentes y marcas podrían ser arbitrables y podrían ser ejecutados. La realidad del arbitraje en nuestro país hace inevitable que este tipo de cuestiones sucedan. 


\section{iii. La necesidad de una decisión erga omnes}

Como se ha mencionado líneas arriba, los derechos sobre patentes y marcas son derechos constitutivos. Es decir, requieren que el Estado otorgue la titularidad de este derecho al particular para poder gozar de protección. En este contexto, una decisión que concluya que la titularidad no fue bien determinada o que fue inválida la concesión de ese derecho, requerirá que se modifique lo estipulado en el registro público de titulares.

En la resolución de una controversia sobre explotación, esta solo afecta la esfera jurídica de las partes. Esto significa que se observará, a partir del contrato el incumplimiento de determinadas cláusulas, el alcance y las condiciones de la cesión de estos derechos. En cambio, las decisiones sobre el otro tipo de controversias sobrepasan el interés de las partes y afecta a terceros. El registro público se hizo con la finalidad de hacer pública la asignación de estas titularidades para evitar el mal uso o el abuso de terceros ${ }^{27}$. Como bien mencionan Julio y Cesar Matheus:

“Dada su falta de existencia corporal, el nacimiento, vida y extinción de la marca descansa en un cuidadoso sistema de publicidad legal, el mismo que le brinda una existencia registral supletoria de su particular naturaleza inmaterial. El derecho a su uso exclusivo se subordina al requisito de su registro ante la oficina competente" 28 .

La lógica detrás de este registro es blindar de seguridad jurídica tanto a los titulares del derecho como a terceros. Entonces, una decisión del tribunal arbitral no podrá cumplir con esta función porque no posee las facultades, como sí las posee Indecopi, de hacer de público conocimiento para el mercado la alteración de este registro.

Nuevamente, las facultades del arbitraje respecto a la ejecución resultan muy limitadas para abarcar las necesidades de los conflictos de Propiedad Industrial, y por lo tanto, terminaría por inutilizarse la vía arbitral.

\section{Conclusión}

En síntesis, la Propiedad Industrial es un área del Derecho que ha sido objeto de diversos cambios a lo largo de los años. Esto ha supuesto que los conflictos que pueden originarse de los derechos de patentes y marcas se han complejizado. El arbitraje ha surgido como respuesta a estas problemática en países como Estados Unidos, Bélgica y Suiza debido a sus múltiples beneficios para la resolución de conflictos de esta índole.

27 MALDONADO, Gabriela. "Propuesta de mediacion y arbitraje en propiedad industrial para la Comunidad Andina de Naciones" (Tesis, Universidad Andina Simón Bolivar, 2001, p. 11.

28 MATHEUS, Julio y MATHEUS, Carlos. "El arbitraje en materia de signos distintivos empresariales”. En Ius et veritas, vol. 28, núm. 1, 2004, p. 266. 
De acuerdo a lo analizado, es jurídicamente posible la arbitrabilidad de todos los conflictos que se generan en estos derechos. Los conflictos originados respecto de la explotación, pueden ser arbitrados por encajar dentro del supuesto de "materia de libre disponibilidad". Por otro lado, a partir de la revisión de doctrina y jurisprudencia internacional, los conflictos que devienen en una determinación de la titularidad y validez de la concesión del derecho podrán ser pasibles de ser sometidos a arbitraje a través de una ley expresa que lo habilite.

Sin embargo, esta posibilidad jurídica no significa una automática conveniencia para un Sistema Jurídico como el peruano. Existen tres motivos principales por los cuales no sería conveniente la arbitrabilidad de la Propiedad Industrial en el Perú: los problemas de ejecución, la complejidad de los criterios aplicables y la necesidad de una ejecución erga omnes.

En primer lugar, porque su ejecución sería dificultosa. Los laudos que resuelvan este tipo de conflictos podrían ser anulados/no reconocidos porque el Poder Judicial siempre puede alegar que se contraviene el orden público por la existencia de normas imperativas que gobiernan alrededor de este derecho. En segundo lugar, existe un alto riesgo de que este tipo de controversias puedan ser revisadas por el Poder Judicial. Esto porque se puede instrumentalizar a la objeción de la existencia del derecho de patente o marca para que los árbitros no puedan tener competencia para pronunciarse sobre la explotación. Si se suma esto a un Poder Judicial que desconoce la solución doctrinal y jurisprudencial del tema, se tiene un riesgo inminente de intromisión de los tribunales nacionales.

En tercer lugar, existirían problemas en los efectos de la decisión arbitral. Cuando esta implique un cambio en la titularidad o en la validez de este derecho, se necesita acompañar esta decisión de un efecto erga omnes. Esto es, una modificación en el registro de inscripción de patentes y marcas. Sin embargo, los tribunales arbitrales no gozan de este tipo de facultades. En cuarto lugar, el siempre peligroso Poder Judicial peruano puede utilizar el argumento de la motivación para anular el laudo. Pese a que no puede revisar el fondo de la controversia, al Poder Judicial poco o nada le ha importado antes este criterio y podría alegar que no se han examinado adecuadamente argumentos jurídicos para la no arbitrabilidad de este tipo de conflictos.

Finalmente, puede concluirse que la arbitrabilidad de la Propiedad Industrial podría estar funcionando en otras legislaciones perfectamente. Sin embargo, debido a la situación del arbitraje peruano, la inclusión de este sería una crónica de muerte anunciada. 